\title{
Avaliação do conhecimento de educadores infantis das escolas municipais frente à avulsão dentária em Patos, Brasil
}

\author{
Fernanda Rocha Mendes SOARES ${ }^{1}$ \\ Ocimar Lopes de OLIVEIRA ${ }^{2}$ \\ Gymenna Maria Tenório GUÊNES ${ }^{3}$ \\ Luanna Abílio Diniz Melquíades de MEDEIROS ${ }^{3}$ \\ Ana Luiza Dias Leite de ANDRADE ${ }^{4}$ \\ Camila Helena Machado da Costa FIGUEIREDO 5
}

Knowledge of children educators about dental avulsion in Patos, Brasil public schools

Conocimiento de educadores infantiles sobre avulsión dental en escuelas públicas de Patos, Brasil

${ }^{I}$ Graduada em Odontologia pela Universidade Federal de Campina Grande, UFCG, Centro de Saúde e Tecnologia Rural, 58700-970, Patos-Paraíba, Brasil. ${ }^{2}$ Graduando em Odontologia pela Universidade Federal de Campina Grande, UFCG, Centro de Saúde e Tecnologia Rural, 58700-970, Patos-Paraíba, Brasil. ${ }^{3}$ Professora Doutora da Unidade Acadêmica de Ciências Biológicas, Centro de Saúde e Tecnologia Rural, Universidade Federal de Campina Grande, Unidade Acadêmica de Ciências Biológicas, Centro de Saúde e Tecnologia Rural, 58700-970, Patos-Paraíba, Brasil.

${ }^{4}$ Professora Doutora do Departamento de Anatomia do Instituto de Ciências Biomédicas da Universidade Federal de Alfenas, UNIFAL/MG, 37130001 - Alfenas, MG, Brasil

${ }^{5}$ Professora Doutora do Curso de Odontologia da Universidade Federal de Campina, UFCG, Centro de Saúde e Tecnologia Rural, 58700-970, Patos-Paraíba, Brasil.

\section{Resumo}

Introdução: A avulsão dentária na infância é bastante frequente e o ambiente escolar é um local susceptível a sua ocorrência em virtude das crianças e pré-adolescentes estarem envolvidas em atividades esportivas. Objetivo: A presente pesquisa teve como propósito avaliar o nível de conhecimento dos educadores do ensino fundamental das escolas municipais de Patos, Paraíba, diante avulsões dentárias no ambiente escolar. Material e Método: Este estudo foi do tipo transversal, observacional, adotando como estratégia de coleta de dados o questionário. Onze escolas municipais de ensino fundamental de Patos foram selecionadas para a nossa pesquisa, assim, a amostra foi composta por 91 professores. O questionário era composto por 15 perguntas, sendo dividido em três partes (caracterização da amostra, experiências prévias e perguntas específicas sobre a avulsão dentária). Resultados: A maioria dos professores é do gênero feminino (87,7\%), com idade entre 20 e 60 anos. Não estão orientados, nem tiveram qualquer tipo de treinamento durante sua formação sobre a avulsão dentária (92\%), nem se consideram preparados para socorrer um aluno $(93,41 \%)$. No caso de uma avulsão, $68,13 \%$ armazenariam o elemento dentário, enquanto 4,39 reposicionaria o dente no seu lugar. Conclusão: Os resultados encontrados nesse estudo demonstram o pouco conhecimento relacionado a procedimentos de urgência a serem realizados em casos de avulsão dentária. Isso indica a grande necessidade de incorporar essa disciplina ao currículo desses profissionais, treinamentos do manejo inicial adequado, além de realizar campanhas educativas em saúde nas escolas, para assim a realidade do prognóstico se tornar positiva.

Descritores: Avulsão Dentária; Conhecimento; Educação Infantil; Professores Escolares.

\section{Abstract}

Dental avulsion in childhood is quite frequent and the school environment is a place susceptible to its occurrence because children and preadolescents are involved in sports activities. The present research had the purpose of evaluating the level of knowledge of the elementary school educators of the municipal schools of Patos, Paraíba, against dental avulsions in the school environment. This cross-sectional, observational study adopted the questionnaire as a data collection strategy. Eleven municipal schools of Patos elementary school were selected for our research, so the sample was composed of 91 teachers. The questionnaire was composed of 15 questions and was divided into three parts (sample characterization, previous experiences and specific questions about dental avulsion). The majority of teachers are female (87.7\%), aged between 20 and 60 years. They are not oriented, nor had any type of training during their training on dental avulsion (92\%), nor do they consider themselves prepared to rescue a student $(93.41 \%)$. In the case of an avulsion, $68.13 \%$ would store the dental element, while 4.39 would reposition the tooth in its place. The results found in this study demonstrate the lack of knowledge related to urgent procedures to be performed in cases of dental avulsion. This indicates the great need to incorporate this discipline into the curriculum of these professionals, training of the appropriate initial management, as well as conducting health education campaigns in schools, so that the reality of the prognosis becomes positive.

Descriptors: Tooth Avulsion; Knowledge; Child Rearing; School Teachers.

\section{Resumen}

La avulsión dental en la infancia es bastante frecuente y el ambiente escolar es un lugar susceptible a su ocurrencia en virtud de los niños y preadolescentes estar involucrados en actividades deportivas. La presente investigación tuvo como propósito evaluar el nivel de conocimiento de los educadores de la enseñanza fundamental de las escuelas municipales de Patos, Paraíba, ante avulsiones dentales en el ambiente escolar. Este estudio fue del tipo transversal, observacional, adoptando como estrategia de recolección de datos el cuestionario. Se seleccionaron once escuelas municipales de enseñanza fundamental de Patos para nuestra investigación, así que la muestra fue compuesta por 91 profesores. El cuestionario se compone de 15 preguntas, dividido en tres partes (caracterización de la muestra, experiencias previas y preguntas específicas sobre la avulsión dental). La mayoría de los profesores son del género femenino (87,7\%), con edad entre 20 y 60 años. No están orientados, ni tuvieron ningún tipo de entrenamiento durante su formación sobre la avulsión dental ( $92 \%$ ), ni se consideran preparados para socorrer a un alumno (93,41\%). En el caso de una avulsión, el 68,13\% almacenar el elemento dental, mientras que 4,39 reposicionaría el diente en su lugar. Los resultados encontrados en este estudio demuestran el poco conocimiento relacionado con procedimientos de urgencia a ser realizados en casos de avulsión dental. Esto indica la gran necesidad de incorporar esta disciplina al currículo de esos profesionales, entrenamientos del manejo inicial adecuado, además de realizar campañas educativas en salud en las escuelas, para así la realidad del pronóstico se vuelva positiva.

Descriptores: Avulsión de Diente; Conocimiento; Crianza del Niño; Maestros.

\section{INTRODUÇÃO}

A avulsão dentária equivale ao total deslocamento do dente do alvéolo, gerando assim lesões como ruptura do feixe vásculo-nervoso apical e das fibras do ligamento periodontal, podendo ocorrer fratura do osso alveolar ${ }^{1}$. A incidência de avulsão dentária varia de 1 a $16 \%$ de todas as lesões traumáticas na dentição permanente, sendo o gênero masculino o mais acometido e a idade prevalente varia de 7 a 11 anos de idade ${ }^{2}$.

A prevalência e frequência de avulsões dentárias são maiores em crianças na idade escolar devido ao desenvolvimento dental incompleto, ao 
periodonto resiliente, e ainda pela frequência da prática de atividades esportivas ou recreativas por essa faixa etária. $\mathrm{O}$ trauma dentário pode comprometer a estética, função mastigatória, fonética, bem como o desenvolvimento mental e social, por isso a participação e conhecimento dos educadores em situações de emergências como avulsão é importante, pois a qualidade dos procedimentos afeta diretamente no prognóstico ${ }^{1,3}$.

Um dente avulsionado pode ser reimplantado, mas dependerá dos cuidados rápidos e apropriados no primeiro momento, que normalmente são fornecidos por profissionais que não são da área de saúde no local do acidente. Desta forma, para o reimplante ser considerado como uma modalidade de tratamento deve-se primar por condições ótimas, como mínima contaminação do elemento dentário avulsionado, o meio de armazenamento extraoral, de preferência, leite, saliva ou solução salina e a rapidez, pois dependendo do tempo pode acontecer reabsorções extensas ${ }^{4}$.

O tratamento de escolha para a avulsão consiste em reimplante do elemento avulsionado realizando a contenção semirrígida por 7 a 14 dias, seguida do tratamento endodôntico de 7 a 10 dias após o seu reimplante e antes da remoção da contenção, além da vacinação contra tétano estar em dia para evitar contaminações e a prescrição de antibiótico. Deve-se considerar que o seu sucesso se deve à ausência de reabsorção radicular e a reparação do ligamento periodontal ${ }^{5}$.

A falta de preparação dos educadores em lidar com situações de avulsão requer bastante atenção, por ser algo de suma importância. Estudos têm mostrado que o conhecimento destes é inadequado, aumentando, assim, possíveis sequelas devido ao não uso das técnicas nos primeiros momentos do acidente $e^{1,6}$.

Com base no exposto, esta pesquisa propõese avaliar o nível de conhecimento dos educadores do ensino fundamental das escolas municipais de Patos, estado da Paraíba, Brasil diante avulsões dentárias no ambiente escolar, afim de minimizar o número de sequelas por falta de atendimento e conduta no primeiro momento do acidente.

\section{MATERIAL E MÉTODO}

Este estudo foi do tipo transversal, observacional, com abordagem indutiva $e$ procedimento comparativo, descritivo, adotando como estratégia de coleta de dados o questionário específico. O universo foi composto por professores de ensino fundamental das escolas públicas de Patos, estado da Paraíba, Brasil.

Atualmente o município apresenta 20 escolas municipais destas, 11 escolas foram selecionadas de forma aleatória para a nossa pesquisa, a Antonio Guedes, Aristides Hand, Firmino Ayres, João
Rodrigues, Manoel Roberto, Maria Chagas, Maria Eudócia, Nelita Nóbrega, Pastor Frank, Sabino Ferreira e Sizenando de Sousa. O município foi selecionado por conveniência em função de ser o de maior porte populacional do Sertão Paraibano e a $3^{\mathrm{a}}$ cidade-pólo do Estado da Paraíba, considerando sua importância socioeconômica.

O município de Patos está localizado no sertão paraibano, distanciando-se da capital (João Pessoa) $298 \mathrm{~km}$ e possuindo 100.732 habitantes. O município, por sua situação geográfica no interior da Paraíba, se constitui num centro polarizador de uma vasta região interiorana do Estado, em torno do qual gravitam 50 municípios, e para o qual convergem os interesses de uma parcela bastante significativa da população. Para este trabalho, selecionou-se a Universidade Federal de Campina Grande instituição de Ensino Superior pública.

Para a participação dos professores nessa pesquisa foram considerados como critérios de inclusão: Ser professor do ensino fundamental de escola pública de Patos, PB; está presente na escola no dia da coleta; Autorização de participação da pesquisa por meio do Termo de Consentimento Livre e Esclarecido (TCLE) assinado. Foram excluídos da pesquisa os professores que apresentassem questionário com preenchimento incompleto

A coleta de dados foi realizada por um pesquisador, através de um questionário estruturado anônimo baseado em estudo prévio 1 contendo questões objetivas para análise do conhecimento dos professores frente à avulsão dentária. Os dados foram coletados nas escolas e só participaram da pesquisa aqueles que estiveram presentes na escola no dia da coleta e assinaram o termo de consentimento livre e esclarecido.

Inicialmente, o convite para participar da pesquisa foi realizado diretamente com a Secretaria de Educação do município de Patos, que assinou a Carta de Anuência, concordando em participar do estudo. Em seguida, os diretores de cada escola foram comunicados sobre a pesquisa e logo após, foi feito o convite aos professores destas escolas. Aqueles que concordaram em participar receberam o TCLE e o questionário da pesquisa por meio impresso. O questionário era composto por 15 perguntas, sendo dividido em três partes: a primeira continha perguntas para a caracterização do professor, como sexo, idade, tempo de trabalho como professor e tempo de trabalho na escola, a segunda parte abordava experiências prévias e se os professores se sentiam preparados para fazer um reimplante frente a um traumatismo dentário, enquanto a terceira foi formada por perguntas específicas sobre a avulsão dentária, a importância do tratamento de emergência e de como agir.

Após coletados, os dados foram registrados na forma de banco de dados do programa de 
informática SPSS (Statistical Package for Social Sciences) para Windows, versão 13.0, e foram trabalhados pela estatística descritiva.

Todos os voluntários participantes da pesquisa preencherão um Termo de Consentimento Livre e Esclarecido, por meio do qual foram informados dos objetivos do estudo e dos benefícios que este poderá trazer à população e também da possibilidade de abandono da pesquisa pelos mesmos em qualquer momento, sem que haja nenhum ônus ao voluntário. Todos os participantes assinaram o termo de consentimento livre e esclarecido, concordando com a participação no presente estudo.

O cálculo amostral considerou um grau de confiança de $95 \%$, poder de teste de $50 \%$ e erro aceitável de 5\%, em um universo de aproximadamente 100 professores, obteve-se uma amostra de 91 participantes. O projeto de pesquisa foi aprovado pelo Comitê de Ética em Pesquisa com Seres Humanos das Faculdades Integradas de Patos (Certificado de Apresentação para Apreciação Ética 90496218.1.0000.5181).

\section{RESULTADOS}

A amostra total foi composta por 91 professores de ensino fundamental das escolas públicas de Patos, Paraíba, sendo $78(87,71 \%)$ do gênero feminino e $13(14,28 \%)$ do gênero masculino. As idades variaram entre 20 e 60 anos com média de 38,8 anos. A Tabela 1 aponta a caracterização da amostra. A maior parte dos professores não está orientada nem teve qualquer tipo de treinamento sobre a avulsão dentária durante sua formação. Igualmente, a maior parte dos professores não se considera preparada para socorrer um aluno (Tabela 2).

Tabela 1. Caracterização da amostra (Patos-PB, Brasil, 2018)

\begin{tabular}{|c|c|c|}
\hline \multirow{2}{*}{ Variáveis } & \multicolumn{2}{|c|}{ Frequência } \\
\hline & $\mathbf{n}$ & $\%$ \\
\hline \multicolumn{3}{|c|}{ Gênero } \\
\hline Feminino & 78 & 85,71 \\
\hline Masculino & 13 & 14,28 \\
\hline \multicolumn{3}{|c|}{ Grau de Formação } \\
\hline Superior incompleto & 04 & 4,39 \\
\hline Superior Completo & 61 & 67,03 \\
\hline Pós-graduação & 26 & 28,57 \\
\hline \multicolumn{3}{|c|}{ Idade (anos) } \\
\hline 20 a 30 & 14 & 15,38 \\
\hline 31 a 40 & 27 & 29,67 \\
\hline 41 a 50 & 41 & 45,05 \\
\hline 51 a 60 & 09 & 9,89 \\
\hline \multicolumn{3}{|c|}{ Tempo de experiência profissional (anos) } \\
\hline $1 \mathrm{a} 10$ & 29 & 31,86 \\
\hline 11 a 20 & 35 & 38,46 \\
\hline 21 a 30 & 22 & 24,17 \\
\hline Não declarou & 05 & 5,49 \\
\hline
\end{tabular}

Tabela 2. Distribuição da amostra sobre formação e treinamento em avulsão dentária dos professores (Patos-PB, Brasil, 2018)

\begin{tabular}{|c|c|c|}
\hline \multirow{2}{*}{ Variáveis } & \multicolumn{2}{|c|}{ Frequência } \\
\hline & $\mathbf{N}$ & $\%$ \\
\hline \multicolumn{3}{|c|}{$\begin{array}{l}\text { Teve algum treinamento de primeiros socorros durante a formação } \\
\text { acadêmica? }\end{array}$} \\
\hline Sim & 25 & 27,47 \\
\hline Não & 66 & 72,53 \\
\hline \multicolumn{3}{|c|}{$\begin{array}{l}\text { Se sua resposta foi sim, a orientação ou treinamento incluiu } \\
\text { informaçôes de como proceder à frente de uma avulsão dentária? }\end{array}$} \\
\hline Sim & 2 & 8 \\
\hline Não & 23 & 92 \\
\hline \multicolumn{3}{|c|}{$\begin{array}{l}\text { Você se considera preparado para socorrer um aluno que sofreu } \\
\text { avulsão dentária? }\end{array}$} \\
\hline Sim & 06 & 6,59 \\
\hline Não & 85 & 93,41 \\
\hline
\end{tabular}

As questões sobre o conhecimento em avulsão dentária por parte dos professores de ensino fundamental das escolas públicas de Patos, Paraíba estão apresentadas na Tabela 3.

Tabela 3: Distribuição da amostra sobre o conhecimento em avulsão dentária dos professores (Patos-PB, Brasil, 2018)

\begin{tabular}{|c|c|c|}
\hline \multirow{2}{*}{ Variáveis } & \multicolumn{2}{|c|}{ Frequência } \\
\hline & $\mathbf{n}$ & $\%$ \\
\hline \multicolumn{3}{|c|}{ O que você faria com o dente que caiu? } \\
\hline Armazenaria o dente & 62 & 68,13 \\
\hline Colocaria dente fora & 13 & 14,28 \\
\hline Não sei & 12 & 13,18 \\
\hline Reposicionaria o dente no seu lugar & 4 & 4,39 \\
\hline \multicolumn{3}{|c|}{$\begin{array}{l}\text { Em sua opinião quanto tempo, o dente pode ficar fora da boca antes de } \\
\text { ser reposicionado? }\end{array}$} \\
\hline Não sei & 70 & 76,92 \\
\hline Imediatamente após o trauma & 7 & 7,69 \\
\hline Até 30 minutos após o trauma & 6 & 6,59 \\
\hline De uma a duas horas após o trauma & 5 & 5,49 \\
\hline $\begin{array}{c}\text { De vinte quatro até sessenta e duas horas após o } \\
\text { trauma }\end{array}$ & 3 & 3,29 \\
\hline \multicolumn{3}{|c|}{ Como você procederia a caso visse no chão um dente avulsionado? } \\
\hline Pegaria o dente pela coroa & 35 & 38,46 \\
\hline Não pegaria o dente & 29 & 31,86 \\
\hline Não sei & 25 & 27,47 \\
\hline Pegaria o dente pela raiz & 2 & 2,19 \\
\hline \multicolumn{3}{|c|}{$\begin{array}{c}\text { Se você não reimplantasse o dente, onde você iria armazena-lo até que o } \\
\text { aluno fosse atendido pelo profissional? }\end{array}$} \\
\hline Envolveria num pedaço de papel, pano ou lenço limpo & 27 & 29,67 \\
\hline Recipiente com líquido & 16 & 17,58 \\
\hline Não sei & 15 & 16,48 \\
\hline Jogaria o dente no lixo & 11 & 12,08 \\
\hline Envolveria numa gaze ou algodão & 08 & 8,79 \\
\hline Deixaria no gelo & 08 & 8,79 \\
\hline Recipiente sem líquido & 06 & 6,59 \\
\hline \multicolumn{3}{|c|}{$\begin{array}{l}\text { Se você assinalou na questão acima que colocaria no líquido, em que } \\
\text { líquido você deixaria o dente? }\end{array}$} \\
\hline Água & 08 & 50 \\
\hline Álcool & 05 & 31,25 \\
\hline Leite fresco & 03 & 18,75 \\
\hline
\end{tabular}

DISCUSSÃO

O ambiente escolar é considerado um local susceptível a ocorrência de traumatismo dentário, uma vez que, as crianças e adolescentes estão envolvidos em atividades esportivas e momentos de recreação. Neste caso, o professor será o responsável pelo primeiro atendimento prestado a criança, sendo assim de fundamental importância que os mesmos tenham conhecimento sobre o trauma dental e o manejo correto dessas crianças em situações emergenciais ${ }^{7}$.

No nosso estudo, evidenciou-se uma deficiência dos professores frente à avulsão dentária, devido à falta de orientação e conhecimento sobre o assunto. A pesquisa apontou que a maior parte dos professores $(72,53 \%)$ não está orientada, nem teve qualquer tipo de treinamento durante sua formação, o que evidencia a necessidade do tema da avulsão dentária ser abordada na disciplina primeiros socorros. O mesmo ocorreu no estudo feito por Hanah e Costa $^{8}$, onde $70,1 \%$ dos professores de ensino fundamental não tiveram qualquer tipo de treinamento.

Segundo Miranda et al. $^{9}$ o sucesso do reimplante dental tem o melhor prognostico quando o implante for realizado nos primeiros trinta minutos da avulsão, o que também é relatado por Maia et al. ${ }^{10}$ que observaram um ótimo prognóstico para reimplante imediato.

Desta forma, o período entre a avulsão do dente e o seu reimplante é considerado de extrema 
importância para o prognóstico do caso, onde o atraso no reimplante reduz drasticamente o sucesso do tratamento, levando à perda do elemento dentário em muitos casos. No presente estudo observou-se que $76,92 \%$ dos professores relataram não saber qual período que o dente avulsionado poderia ficar fora do alvéolo. O mesmo foi observado no estudo de Manegotto et al. ${ }^{1}$, onde $59,9 \%$ dos professores afirmaram não saber o período correto de reimplante dentário, assim é possível identificar a falta da conduta correta diante uma urgência de avulsão dentária no ambiente escolar.

Ainda no nosso estudo, os educadores foram questionados sobre o tipo de armazenamento para ser transportado o dente avulsionado até o dentista sem qualquer dano. Assim, uma grande variedade de respostas foi encontrada, onde pedaço de papel, pano, algodão, armazenamento com liquido e sem liquido foram respostas frequentes.

Dentre os professores que responderam que armazenariam em algum líquido, temos o leite como uma das melhores opções de transporte, contudo, esta opção obteve a menor porcentagem de respostas, onde apenas $18,75 \%$ optaram por essa alternativa. No estudo feito por Carylofo et al. ${ }^{3}$, apenas $13,1 \%$ dos educadores optariam pelo leite como melhor escolha.

Segundo conclusões do estudo de Wang et al. ${ }^{11}$, o leite possui um ótimo efeito na viabilidade e diferenciação osteogênica nas células troncos do ligamento periodontal.

Quando questionados sobre o que fazer partindo da hipótese do dente ter caído no chão, $38,46 \%$ pegariam o dente pela coroa, $2,19 \%$ pela raiz, $31,86 \%$ não pegaria o dente e $27,47 \%$ não sabia o que fazer. De acordo com Andersson et al. ${ }^{12}$ a melhor forma de manipular o dente é pela coroa, o manejo incorreto do órgão dentário pode causar possíveis danos as células periodontais. Logo nota-se nesta questão a falta de informação e despreparo em sintonia com os resultados em outros estudos.

Conscientizados da falta de preparação e orientação sobre a conduta adequada frente a casos de avulsão, a maioria dos professores $(93,41 \%)$ não se consideram capacitados para socorrer um aluno que sofreu um tipo de traumatismo, dificultando seu tratamento e prognóstico.

CONCLUSÃO

Os resultados encontrados nesse estudo demonstram o pouco conhecimento relacionado a procedimentos de urgência a serem realizados em casos de avulsão dentária. Isso indica a grande necessidade de incorporar essa disciplina ao currículo desses profissionais, treinamentos do manejo inicial adequado, além de realizar campanhas educativas em saúde nas escolas, para assim a realidade do prognóstico se tornar positiva.

\section{REFERÊNCIAS}

1. Menegotto A, Scatena C, Pereira JT, Werle SB, Oliveira RS. Avaliação do conhecimento dos professores de escolas públicas quanto ao manejo da avulsão dentária em crianças. Perspectiva: Ciência e Saúde. 2017;2(1):83-94

2. Mota Junior CR, Silva TPC. Avulsão dental em dentes permanentes. Rev cient ITPAC. 2009;2(2):8-23.

3. Curylofo PA, Lorencetti KT, Silva SRC. Avaliação do conhecimento de professores sobre avulsão dentária. Arq Odontol. 2012;48(3):175-80.

4. Poluha LR, Nascimento GHH, Terada HH. Reimplante de dentes decíduos: indicações e contra-indicações. Arch Health Invest. 2016;5(3):140-43.

5. Rebouças PD, Moreira JJS, Souza DL. Fatores que influenciam no sucesso do reimplante dental. Publ UEPG. Ci Biol Saúde. 2013;19(1):31-7.

6. Costa LED, Queiroz FS, Nobrega CBC, Leite MS, Nobrega WFS, Almeida ER. Dental trauma in childhood: evaluation action of educators in public nurseries from the city of Patos/PB. Rev odontol UNESP. 2014;43(6):402-8.

7. Bittencourt AM, Pessoa OF, Silva JM. Avaliação do conhecimento de professores em relação ao manejo da avulsão dentária em crianças. Rev odontol UNESP. 2008; 37(1):15-9.

8. Hanah SA, Costa SK. Conhecimento dos professores frente a avulsão dentária. Pesq Bras Odontoped Clin Integr. 2010;10(1):27-33.

9. Miranda ACE, Habitante SN, Candelária LFA. Revisão de determinados fatores que influenciam no sucesso do reimplante dental. Rev biociênc Taubaté.2000;6(1):35-9.

10. Maia SMA, Travassos RMC, Mariz EB, Macêdo SM, Alencar TA. Conduta clínica do cirurgiãodentista ante a avulsão dental: revisão de literatura. RSBO. 2006;3(1):41-7.

11. Wang WJ, Zhao YM, Feng XY, Jia WQ, Ge LH. Effect of skimmed pasteurized milk and Hank's balanced salt solution on viability and osteogenic differentiation of human periodontal ligament stem cells. Dent Traumatol. 2013;29(5):365-71.

12. Andersson L, Andreasen JO, Day P, Heithersay $\mathrm{G}$, Trope M, Diangelis AJ et al. International Association of Dental Traumatology guidelines for the management of traumatic dental injuries: 2. Avulsion of permanent teeth. Dent Traumatol. 2012;28(2):88-96. 


\section{CONFLITO DE INTERESSES}

Os autores declaram não haver conflitos de interesse.

\section{AUTOR PARA CORRESPONDÊNCIA}

Camila Helena Machado da Costa Figueiredo

Avenida dos Universitários, S/N, Rodovia Patos/Teixeira, km1, Jatobá, CEP: 58700-970, Patos, Paraíba, Brasil

E-mail: camila_helena_@hotmail.com

Submetido em 25/06/2019

Aceito em 20/04/2020 